\title{
Atributos INDIVIDUAIS, BACKGROUND FAMILIAR E AS CHANCES DE SUCESSO DOS Candidatos ao Vestibular da UFC
}

Daniel Barboza Guimarães Daitoradbemandamento enEcanomia pda UnivesidadeFedaral do Ceará eProfessor Assistenteda Universidade Feedaral do Ceará barbozadan@hotmail

\section{Ronaldo de Albuquerque e Arraes}

Pós-Dattarado pada Univesity of Tennesse System Direcãa eAdministraçãodo Curso dePós Graduação emEconomia- UFC ronald@ufchr

\section{RESUMO}

Analisar os determinantes dos desempenhos de candidatos ao concurso vestibular da UFC constitui-se o foco central deste artigo. A metodologia baseou-se em uma função de produção de rendimento educacional e estimação feita por modelos econométricos de escolha discreta, dicotômico e policotômico, com o fito de se extrair as chances de os candidatos lograrem êxito em todas as áreas do concurso. Para tanto, foi utilizada uma base de dados derivada de questionários socioeconômicos dos candidatos, e disponibilizados pela Pró-Reitoria de Graduação da UFC. D os resultados estatisticamente robustos, constatou-se no vetor de variáveis socioeconômicas um forte efeito negativo na chance de sucesso no vestibular para aqueles candidatos que se encontram no mercado de trabalho. Bakkgaundfamiliar foi incisivo sobre os sucessos dos candidatos, vez que aqueles que possuem pais com nível superior demonstram possuir melhor performance no exame. A qualidade do ensino público de nível médio transpareceu nos resultados ao reduzir sobremaneira as chances de êxito para os candidatos advindos de escolas públicas. Analisando-se os desempenhos por área, concluiu-se, dentro de cenários de previsão, que candidatos com elevada chance de sucesso em determinada área não a manteria em outras áreas. D escartou-se o efeito concorrência como determinante do desempenho em cada área.

Palavras-chave: Vestibular da UFC. A tributos dos Candidatos. D eterminantes do Sucesso.
Analyzing thepeformancedteminants of candidatestudantsin the college entrancexamination at UFC is themain djetive of this artide Themthoddogy framevork is based upon thespeification of a retum to ecturation production function and its estimation is done through economenicmodls of dismetedhice, didhotomous and podydotomous with theaimof extradingshdent's dhanes to achiere sucess in theexaminationin all areas of undergradatecourses offered by theunivesity. Thus a databasedaived from thecandidates' soio economicsurves was madeavailable by the UndergadrateOffice of UFC. From thestatisticallyrdaust results it vas veified in thesodio economic variables vetor a strongnegativeeffet in the hance of surcess in the examination for thosecandidates that areengaged in thelabor marke. Family badkground wasinisiveon candidates' surcesses, since those that have parents with odlege eduration demonstrateto attain better pefommen in theexamination Thequality of puldicsecondary schoding vas refleted in theresults by reduringsigificantly the hanres of sucess for studants frompuldicshods Foreast semarios within areas of knoweelgeallowed infering that candidates with high hance of sucessin cetain ares would not maintain it in others Thecompe tition effeet was discarded as deisivefactor of peformanrein @ich area.

Keywords: CollegeEntranceExamat UFC. Candidatés Badkgraind Success Determinants 


\section{INTRODUÇÃO}

A educação é tida tanto como um dos mecanismos mais importantes que determinam o processo de mobilidade social entre as gerações (DUMAS; LAMBERT, 2007) como um dos fatores cruciais para ditar a força motriz do crescimento, juntamente com a criação de inovações tecnológicas, na geração de externalidades para prover rendimentos crescentes de produtividade no longo prazo (ROMER, 1986; LUCAS, 1988).

Muitos estudos presentes na literatura internacional vigente já reconhecem a importância da educação para o desenvolvimento tecnológico de uma nação. Em um desses estudos, Freeman (apud AVENA, 2003) mostrou a importância da educação de nível superior para o rápido crescimento de um país, como ocorreu com a Coreia do Sul, que após intensificar os investimentos em capital humano passou de um país subdesenvolvido para uma potência econômica mundial. Além das contribuições econômicas, a educação também está sendo associada a uma variedade de benefícios, como melhorias na saúde, redução da criminalidade, maior coesão social e participação democrática (DE FELÍCIO ; FE RNAND ES, 2005).

No Brasil, embora seja extensa a quantidade de trabalhos realizados na área de educação, poucos se dedicaram a analisar os fatores determinantes das performances dos estudantes. Soares (2004), por exemplo, buscou analisar o desempenho cognitivo de estudantes do ensino fundamental, constatando haver grandes diferenças nos resultados entre as regiões do País, ao levar em consideração fatores socioeconômicos, como raça, diferentes posições sociais e gêneros. Ao notar que a grande maioria dos estudantes tem desempenho menor que o esperado para sua série, conclui que se verificam problemas de qualidade e equidade na educação básica brasileira. Nesse mesmo estudo, o autor identificou variáveis que influenciam o nível de desempenho dos alunos de 8aㅗ série do ensino fundamental, mas não encontrou variáveis que possam diminuir as diferenças de desempenho nos grupos definidos por cor e nível socioeconômico. Em estudo correlato, porém, buscando identificar alguns determinantes das divergências na efetividade dos rendimentos dos alunos nas escolas de diferentes redes de ensino, Costa e Arraes (2006) concluíram que as escolas privadas e em regiões mais desenvolvidas proporcionam melhores condições ao desenvolvimento educacional e, consequentemente, ao desempenho dos estudantes.

Quanto ao ensino superior brasileiro, Velloso (2006) utilizou dados de um censo do Distrito Federal para testar a adequação do agrupamento dos cursos adotados no estudo como um substituto aproximado do nível socioeconômico dos candidatos. Constatou que a renda média familiar dos aprovados cai, à medida que se passa dos grupos de maior para os de menor prestígio, em termos de concorrência.

Para complementar a constatação de Velloso, Queiroz (2008) utilizou dados da Universidade Federal da Bahia e observou que os cursos de mais alta seletividade socioeconômica, que são também os de maior concorrência, são frequentados por estudantes de mais elevado desempenho. A autora também observou que os estudantes oriundos da escola privada apresentaram melhor desempenho no vesti- bular que os originários da escola pública, e que a formação de nível superior dos pais tem efeito decisivo sobre o desempenho do estudante.

Seguindo uma abordagem direcionada ao tipo de escola, D uczmal e Pereira (2003) realizaram uma análise da correlação entre o desempenho no vestibular de escolas particulares de Belo Horizonte e o valor das mensalidades cobradas. Concluíram que existe realmente uma correlação significativa entre esses dois fatores, refletindo assim a percepção do mercado em relação ao desempenho das escolas no vestibular de universidades públicas.

Um resultado bastante interessante, e até o momento inédito, foi alcançado por G uimarães (2007). Utilizando dados do vestibular da Universidade de São Paulo (USP), concluiu que estudantes negros apresentam desempenho inferior aos brancos em proporção maior que a pior performance dos alunos de escola pública em relação aos estudantes de escola particular.

$\mathrm{Na}$ tentativa de se estimar os determinantes chaves da performance dos estudantes no vestibular da Universidade Federal de Pernambuco (UFPE), G uimarães e Sampaio (2007) utilizaram dados de características pessoais dos estudantes, tais como idade, gênero, raça, religião, entre outros. Fazendo uso de regressão quantílica, os autores encontraram alguns resultados já esperados em acordo com a literatura, como 0 caso das variáveis renda familiar e nível de escolaridade dos pais, que apresentaram impactos positivos no desempenho dos estudantes no vestibular da UFPE, principalmente quando se analisa os quantis superiores.

0 enfoque principal do presente trabalho é verificar 0 impacto que algumas variáveis socioeconômicas exercem no desempenho dos candidatos inscritos no vestibular da Universidade Federal do Ceará (UFC), no ano de 2004. E a partir daí, construir cenários probabilísticos sobre os perfis dos candidatos com maiores e menores chances de obter sucesso no exame, por área de concentração, e entre os aprovados para a segunda fase.

Na próxima seção faz-se destaque para a evolução da concorrência no vestibular, seguida de um detalhamento da estrutura metodológica, baseada em modelos de escolha discreta, os quais permitirão expor os resultados probabilísticos por meio de cenários dos perfis dos candidatos com maiores e menores chances de obter êxito. Em seguida, finaliza-se 0 trabalho com as principais conclusões.

\section{CONCORRÊNCIA NO VESTIBULAR}

Nas últimas décadas, a educação superior vem ganhando grande destaque entre os pesquisadores. Isso decorre do fato de que no último meio século, aproximadamente, a demanda por esse nível de educação vem crescendo a taxas expressivas, principalmente nos países desenvolvidos, fazendo do ensino superior uma concentração de elites, acessível em grande parte aos grupos mais ricos e privilegiados (KAPUR; CROWLEY, 2008).

Mesmo com o grande aumento no número de vagas destinadas ao ensino superior, o que se nota é que essa ampliação não foi acompanhada de um processo de democrati- 
zação no acesso à universidade. Para Brito e Carvalho (apud QUEIROZ, p. 1), "Esse processo, ao contrário de democratizar oportunidades, parece ter mantido e até mesmo aprofundado a seletividade que marca esse nível do sistema de ensino". Além da seletividade no acesso ao ensino superior, outro grave problema da atualidade é que a educação superior é cada vez mais importante para a inserção do indivíduo no mercado de trabalho, visto que, em média, os rendimentos dos indivíduos com curso superior são mais elevados (AVENA, 2003). Com isso, nota-se que a demanda pelo ensino superior vem crescendo sistematicamente ao longo dos anos, tornando-se bem maior que a oferta. 0 gráfico abaixo mostra a evolução do número de inscrições em instituições de ensino superior nos últimos anos.
Nota-se que as instituições particulares vêm ganhando mais espaço no mercado do ensino superior, mas principalmente pode-se aferir pelo $\mathrm{G}$ ráfico 1 que as instituições públicas, federais e estaduais ainda são bastante demandadas pelos brasileiros. Os Gráficos 2 e 3 mostram a evolução da concorrência por vagas e por Instituições nos últimos anos.

0 G ráfico 2 mostra a relação entre candidatos inscritos por vaga, e 0 Gráfico 3, a relação entre candidatos inscritos pelo número de instituições de ensino superior. Pode-se constatar que, apesar do aumento do número de instituições federais e estaduais de ensino superior bem como das vagas e inscrições nessas instituições, a concorrência vem crescendo bastante, indicando que a demanda está aumentando a taxas bem maiores que a oferta. Q uanto às instituições privadas, que vêm

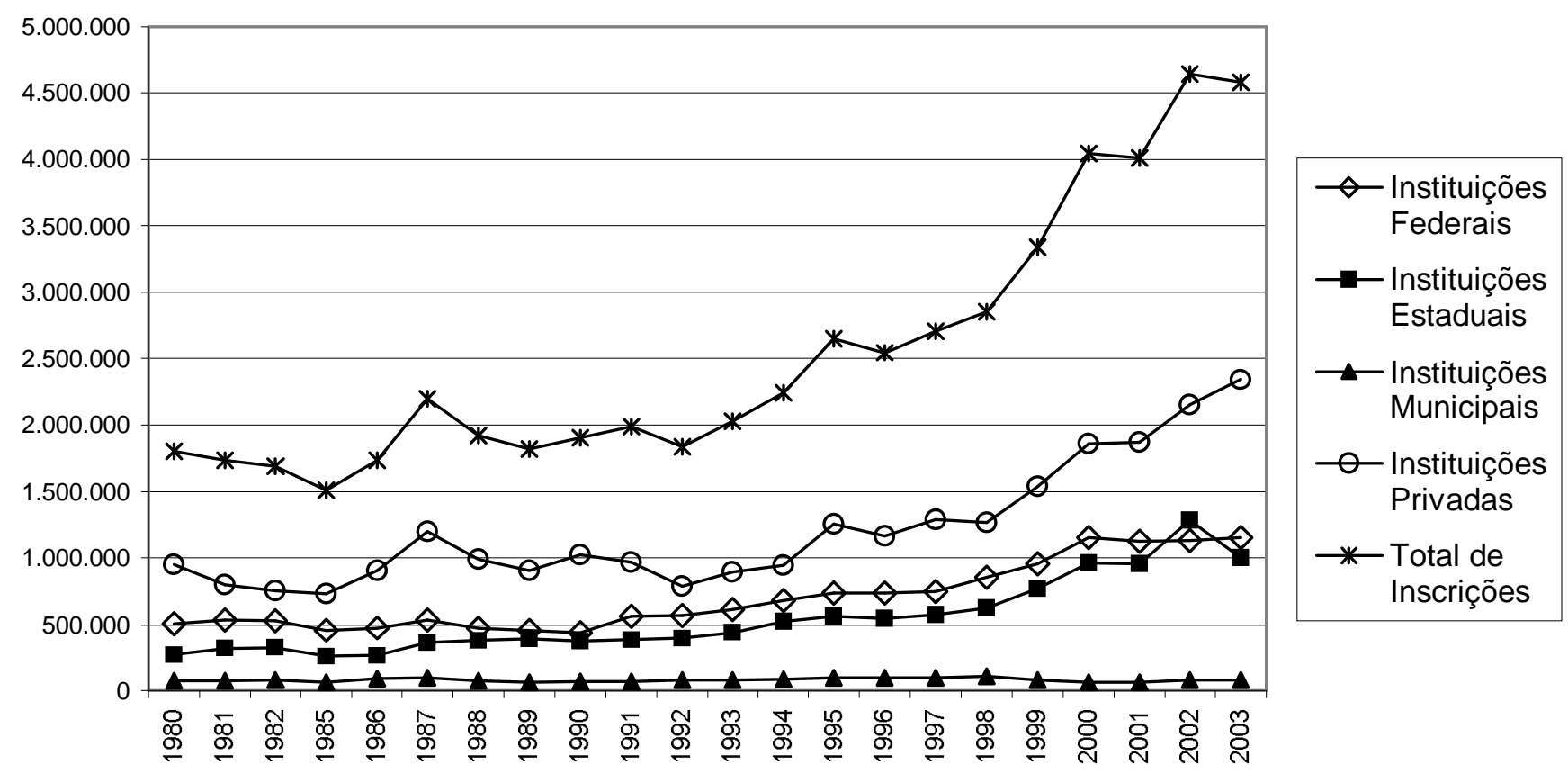

Grafico 1: Evolução do Número de Inscrições no Vestibular no Brasil (1980-2003)

Fonte: MEC/INEP/ SEEC.

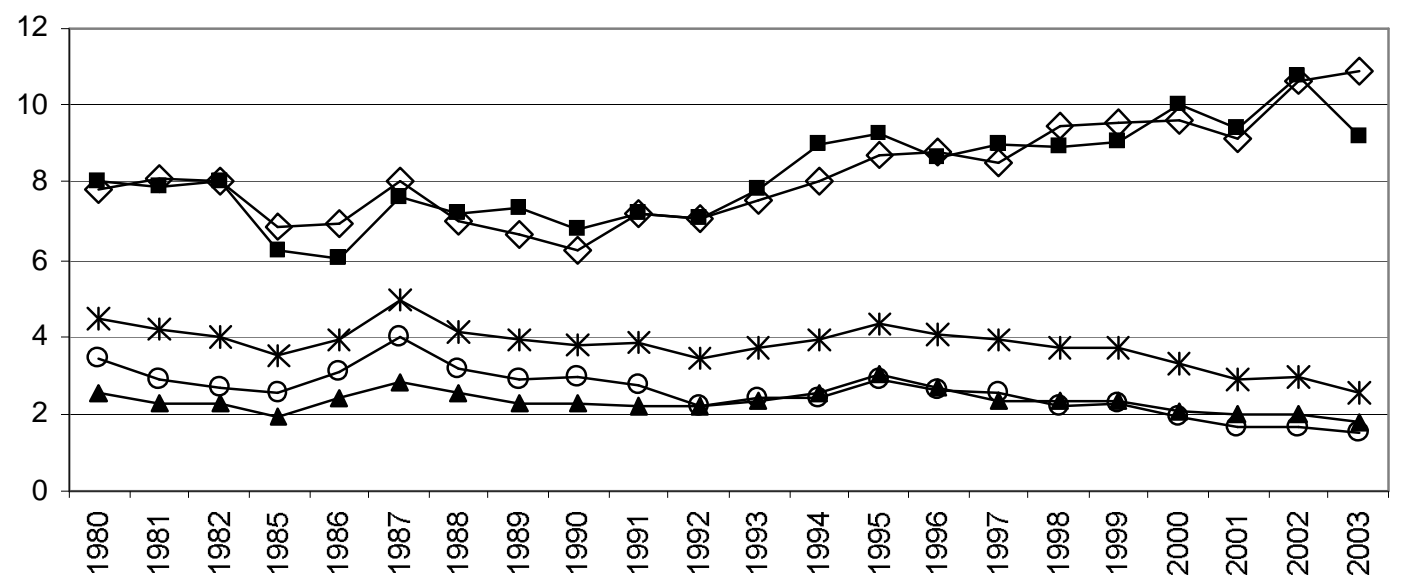
$\diamond$ Instituições Federais
$\rightarrow$ - Instituições Estaduais
$\multimap$ Instituições Municipais
- Instituições Privadas
* Total de Instituições

Grafico 2: Concorrência: Candidatos por Vaga nas Instituições de Ensino Superior no Brasil (1980-2003)

Fonte: MEC/INEP/ SEEC. 
respondendo por grande parte da evolução do ensino superior no Brasil, percebe-se que em relação às vagas há uma queda na concorrência e em relação às instituições a situação está praticamente estabilizada. As instituições municipais se comportaram de maneira similar às instituições privadas.
A variável dependente assumirá um comportamento binário e multinomial ordenado. Na próxima seção, será feita uma abordagem dos dois modelos utilizados neste trabalho, bem como das suas respectivas variáveis dependentes.

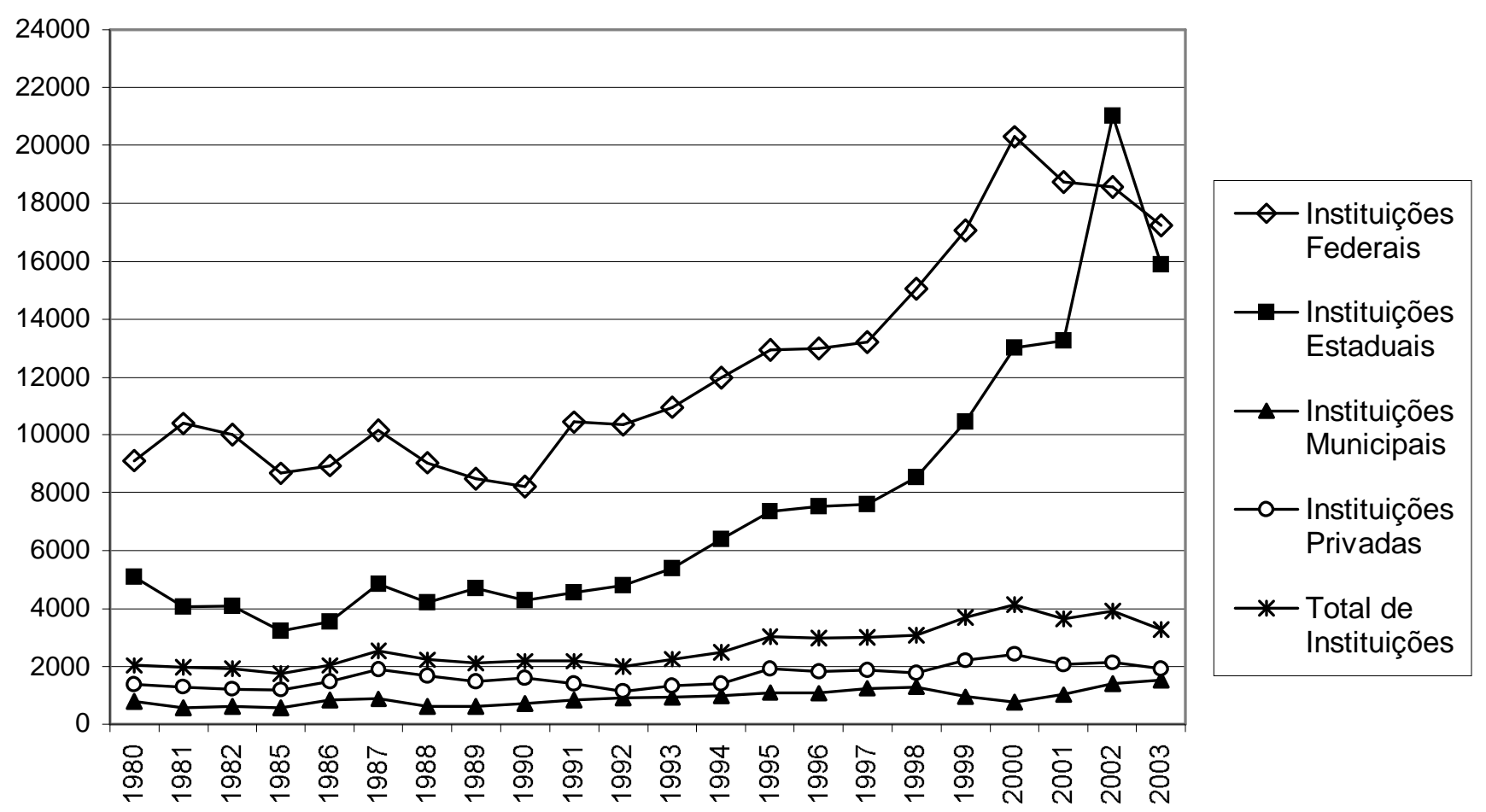

Grafico 3: Concorrência: Candidatos por Instituições de Ensino Superior no Brasil (1980-2003)

Fonte: MEC/ INEP/ SEEC.

\section{METODOLOGIA}

Em modelos econômicos, cujo fenômeno a ser explicado é do tipo qualitativo, utilizam-se variáveis de escolha discreta para medi-lo e, dependendo da extensão do problema investigado, variáveis dicotômicas ou policotômicas são utilizadas. Uma vez que no presente trabalho serão aferidos os determinantes de desempenho no vestibular com enfoques variados, ambos os tipos de variáveis serão utilizadas. No primeiro tipo, será aplicado um modelo dicotômico, e, no segundo, um multinomial ordenado, ambos com a hipótese Probit.

\subsection{Descrição dos Dados}

Os dados utilizados nos modelos foram cedidos pela Comissão Coordenadora do Vestibular (CCV) da UFC, referentes ao vestibular do ano de 2004, cujas informações constam no Questionário Socioeconômico respondido pelo próprio aluno no ato da inscrição para o concurso vestibular. No questionário, são respondidas perguntas do tipo de múltipla escolha referentes a atributos individuais e educacionais dos candidatos, bem como seu status e badkgrand familiar. 0 Q uadro 1 sintetiza as variáveis compiladas dos questionários relativas ao candidato, e que serão utilizadas nas estimações dos modelos, bem como a expectativa de seus efeitos sobre as chances de obtenção de êxito.

\begin{tabular}{|c|l|c|}
\hline $\begin{array}{c}\text { Variáveis } \\
\text { Explicativas }\end{array}$ & \multicolumn{1}{|c|}{ Descrição } & Expectativa \\
\hline Ensino Médio & $\begin{array}{l}\text { 1, se estudou em } \\
\text { escola privada } \\
\text { 0, caso contrário }\end{array}$ & positivo \\
\hline $\begin{array}{c}\text { Número de Vezes } \\
\text { que Prestou } \\
\text { Vestibular }\end{array}$ & $\begin{array}{l}\text { 1, se uma ou mais } \\
\text { vezes }\end{array}$ & positivo \\
\hline Participação na contrário & 1, se trabalha & negativo \\
Vida E conômica & $\begin{array}{l}\text { 0, caso contrário } \\
\text { da Família }\end{array}$ & \\
\hline Nível de & $\begin{array}{l}\text { 1, se possui nível } \\
\text { superior } \\
\text { 0, caso contrário }\end{array}$ & positivo \\
\hline Instrução do Pai & $\begin{array}{l}\text { 1, se possui nível } \\
\text { superior } \\
\text { 0, caso contrário }\end{array}$ & positivo \\
\hline Nível de & \\
\hline Instrução da Mãe
\end{tabular}

Quadro 1: D escrição das Variáveis Explicativas e Expectativa de seus Efeitos

Fonte: Elaborado pelos autores, com base nos dados da CCV.

\subsection{Modelos Econométricos}

Hanushek (1986) argumenta que as pesquisas econômicas de natureza empírica em escolaridade devem se iniciar 
com um modelo conceitual do processo educacional. Segundo 0 autor, um ponto de partida natural são os modelos econômicos da teoria de produção e comportamento da firma. Na mesma linha, outros autores, tais como Albernaz et al (2002), Costa e Arraes (2006), utilizaram-se do que ficou co-nhecido como "função de produção educacional", determinada pelos insumos cumulativos dos alunos, das famílias, das escolas e dos professores. A especificação econométrica dessa função assume a forma geral dada por $Y=X^{\prime} \beta+\varepsilon$, onde $Y$ denota o desempenho dos alunos, que é função de fatores explicativos representados por $X$. Assim, a partir da estimação dessa equação, ter-se-á embasamento para se processar inferências estatísticas, com a finalidade de se avaliar o efeito que as mudanças ocorridas nas variáveis socioeconômicas escolhidas para o presente estudo provocarão no desempenho dos candidatos do vestibular da UFC. Para se alcançar essa finalidade, serão adotados modelos de escolha discreta, dicotômico e policotômico, os quais são detalhados nas subseções seguintes.

\subsubsection{Modelo Dicotômico}

Esse modelo é desenvolvido por meio do uso de uma variável não observada denominada latente, por substituir uma variável binária, a qual assumidamente possui determinada probabilidade de ocorrência (DAVID SO N ; MACKINNO N, 2004). Nesses termos, a especificação geral do modelo é dada por $Y_{\mathrm{i}}=X^{\prime}{ }_{\mathrm{i}} \beta+\mu_{\mathrm{i}}$, onde $Y_{\mathrm{i}}=1$, se ocorre sucesso ${ }^{1}$, ou $Y_{\mathrm{i}}=0$ caso contrário. Como a estimação desse modelo não garante $Y_{\mathrm{i}}$ estar contida no intervalo $(0,1)$, a reformulação do modelo é dada pela criação de uma variável latente $\left(Y_{\mathrm{i}}{ }^{0}\right)$ em substituição a $Y_{\mathrm{i}}$. Ao assumir-se hipótese Probit, onde $\mu_{\mathrm{i}}$ segue uma distribuição normal padronizada, o sinal da variável latente não observada $\left(Y_{i}^{0}\right)$ determinará o valor que a variável binária observada $\left(Y_{\mathrm{i}}\right)$ assumirá, ou seja, se $Y_{\mathrm{i}}^{0}$ for positiva ou negativa, correspondentemente $Y_{\mathrm{i}}$ assume 0 valor 1 ou 0 . D esse modo, torna-se possível computar a probabilidade de que $Y_{\mathrm{i}}$ assuma 0 valor 1, a qual será dada por:

$$
\begin{gathered}
\operatorname{Pr}\left(Y_{\mathrm{i}}=1\right)=\operatorname{Pr}\left(Y_{\mathrm{i}}^{0}>0\right)=\operatorname{Pr}\left(X_{\mathrm{i}}^{\prime} \beta+\mu_{\mathrm{i}}>0\right)=\operatorname{Pr}\left(\mu_{\mathrm{i}}>0-X^{\prime}{ }_{\mathrm{i}} \beta\right)= \\
=\operatorname{Pr}\left(\mu_{\mathrm{i}}<X_{\mathrm{i}} \beta\right)=\Phi\left(X_{\mathrm{i}}^{\prime} \beta\right)
\end{gathered}
$$

Onde $\Phi\left(X^{\prime}{ }_{\mathrm{i}} \beta\right)$ é a função de probabilidade cumulativa da distribuição normal.

Para a finalidade deste trabalho, ocorrerá o sucesso da variável binária $\left(Y_{i}=1\right)$, se a nota do candidato ( mota $)$ for maior do que a média das notas da área do conhecimento na qual ele se inscreveu; consequentemente, assumirá 0 valor 0 , caso contrário. As nove áreas selecionadas serão denotadas por centros ou faculdades, a saber: Centro de Ciências Agrárias; Centro de Ciências; Faculdade de D ireito; Faculdade de Educação; Faculdade de E conomia, Administração, A tuária, Contabilidade e Secretariado; Centro de Humanidades; Faculdade de Medicina; Faculdade de O dontologia, Farmácia e Enfermagem; Centro de Tecnologia. Portanto, haverá nove equações a ser estimadas, cada uma delas representando uma área do conhecimento, em que as variáveis explicativas estão descritas no Quadro 1, e a variável dependente dada por:
$Y_{\mathrm{i}}=\left\{\begin{array}{l}1, \text { se a nota do candidato for maior que a média de sua } \\ 0, \text { caso contrário }\end{array}\right.$

\subsubsection{Modelo Policotômico}

Do mesmo modo que a especificação dicotômica da seção anterior, o modelo Policotômico do tipo ordenado também poderá ser especificado por meio do uso de uma variável latente, com a flexibilização de que a variável dependente poderá assumir um número de valores discretos maior do que dois (caso dicotômico), tendo a seguinte formatação: $Y_{\mathrm{i}}=\boldsymbol{j}$ se $\theta_{\mathrm{j}-\mathbf{1}}<Y_{\mathrm{i}}^{0}<\theta_{\mathrm{j}}$. O s limites definidos por $\theta_{\mathrm{j}}$ são denominados parâmetros threshdd, os quais serão determinados a priori em função da nota máxima possível de ser obtida na primeira fase do vestibular, que é 240 pontos. D essa forma, definiu-se a ordenação em três grupos distribuídos igualmente com $1 / 3$ em cada um deles, resultando nas seguintes especificações das variáveis discreta e latente:

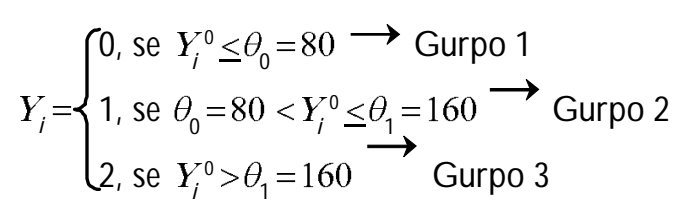

Similarmente ao modelo binário, nesse também serão calculadas as probabilidades para que a variável dependente assuma um dos três valores, diferenciando pela dependência dos parâmetros threshold As probabilidades para a ocorrência de $Y_{\mathrm{i}}=\boldsymbol{j}$ serão dadas por:

$$
\begin{aligned}
& \operatorname{Pr}\left(Y_{\mathrm{i}}=0\right)=\operatorname{Pr}\left(Y_{\mathrm{i}}^{0} \leq \theta_{1}\right)=\operatorname{Pr}\left(X^{\prime}{ }_{\mathrm{i}} \beta+\mu_{\mathrm{i}} \leq \theta_{1}\right)=\operatorname{Pr}\left(\mu_{\mathrm{i}} \leq \theta_{1}-X^{\prime}{ }_{\mathrm{i}}\right. \\
& \beta)=\Phi\left(\theta_{1}-X^{\prime} \beta\right) \\
& \operatorname{Pr}\left(Y_{\mathrm{i}}=2\right)=\operatorname{Pr}\left(Y_{\mathrm{i}}{ }^{0} \leq \theta_{2}\right)=\operatorname{Pr}\left(X_{\mathrm{i}}^{\prime} \beta+\mu_{\mathrm{i}} \leq \theta_{2}\right)=\operatorname{Pr}\left(\mu_{\mathrm{i}} \leq \theta_{2}-X_{\mathrm{i}_{\mathrm{i}}}\right. \\
& \beta)=\Phi\left(X_{\mathrm{i}^{\prime}} \beta-\theta_{2}\right) \\
& \operatorname{Pr}\left(Y_{\mathrm{i}}=1\right)=1-\operatorname{Pr}\left(Y_{\mathrm{i}}=2\right)-\operatorname{Pr}\left(Y_{\mathrm{i}}=0\right)
\end{aligned}
$$

\section{RESULTAD OS}

Os resultados das estimativas advindas das duas abordagens descritas na seção anterior permitirão inferir sobre as chances dos candidatos a determinados desempenhos no vestibular da UFC por área, com base em suas classificações na primeira etapa em cada área².

\subsection{Resultados do Modelo Dicotômico}

De acordo com as tabelas em anexo, verificou-se que todas as equações mostraram-se estatisticamente significantes, apoiadas nos testes da razão de verossimilhança, que apresentou valores-p próximos a zero nas nove equações estimadas, um para cada área, implicando que se pode rejeitar a hipótese de que todos os coeficientes sejam nulos. Além do mais, todos os coeficientes, em todas as equações, mostraram-se individualmente significantes e de acordo com o sinal esperado (Tabelas A1 a A9 no anexo). 
Uma vez que todas as variáveis explicativas são binárias, comparando-se então dois candidatos com os mesmos atributos, exceto que um não trabalha e o outro trabalha, isso implicará que o segundo apresentará uma menor probabilidade de obter um rendimento de nota superior ao rendimento médio, independentemente da área na qual esses dois indivíduos se encontrem. Para o restante das demais variáveis, que possuem efeito positivo sobre a performance do candidato, o raciocínio é feito de maneira análoga, porém em sen- tido inverso. A Tabela 1 apresenta alguns cenários relativos aos desempenhos dos candidatos ao vestibular, nos quais são denotadas alternâncias de atributos de uma ou mais variáveis. Cabe notar que em todos os cenários, por cada área, há dois referenciais comuns. No primeiro, todas as variáveis assumem 0 valor zero e em seguida alternam-se as variações dos atributos. No segundo, apenas a variável ensino médio é diferente de zero. A linha pontilhada na Tabela faz a distinção dos dois referenciais.

Tabela 1: Cenários para o D esempenho no Vestibular, por Centros e Faculdades

\begin{tabular}{|c|c|c|c|c|c|c|}
\hline Área & Ensino Médio & No de Vezes & Trabalho & Instrução do Pai & Instrução da Mãe & Probabilidades (\%) \\
\hline \multirow{9}{*}{$\begin{array}{l}\text { Centro de } \\
\text { Ciências } \\
\text { Agrárias }\end{array}$} & 0 & 0 & 0 & 0 & 0 & 17,74 \\
\hline & 0 & 0 & 1 & 0 & 0 & 14,30 \\
\hline & 0 & 1 & 0 & 0 & 0 & 35,78 \\
\hline & 0 & 0 & 0 & 1 & 0 & 23,13 \\
\hline & 0 & 0 & 0 & 0 & 1 & 23,54 \\
\hline & $--\overline{1}$ & 0 & -0 & 0 & 0 & $--\overline{32}, 4 \overline{0}=-$ \\
\hline & 1 & 0 & 0 & 1 & 0 & 39,52 \\
\hline & 1 & 0 & 0 & 0 & 1 & 40,04 \\
\hline & 1 & 0 & 0 & 1 & 1 & 47,55 \\
\hline \multirow{9}{*}{$\begin{array}{l}\text { Centro de } \\
\text { Ciências }\end{array}$} & $\overline{0}$ & 0 & 0 & 0 & 0 & 44,97 \\
\hline & 0 & 0 & 1 & 0 & 0 & 41,14 \\
\hline & 0 & 1 & 0 & 0 & 0 & 64,76 \\
\hline & 0 & 0 & 0 & 1 & 0 & 52,09 \\
\hline & $-\mathbf{0}$ & 0 & 0 & 0 & 1 & 58,16 \\
\hline & & & & & & 65,31 \\
\hline & 1 & 0 & 0 & 1 & 0 & 71,65 \\
\hline & 1 & 0 & 0 & 0 & 1 & 76,62 \\
\hline & 1 & 0 & 0 & 1 & 1 & 81,72 \\
\hline \multirow{9}{*}{$\begin{array}{c}\text { Faculdade de } \\
\text { Direito }\end{array}$} & 0 & 0 & 0 & 0 & 0 & 5,26 \\
\hline & 0 & 0 & 1 & 0 & 0 & 3,44 \\
\hline & 0 & 1 & 0 & 0 & 0 & 14,51 \\
\hline & 0 & 0 & 0 & 1 & 0 & 11,11 \\
\hline & 0 & 0 & 0 & 0 & 1 & 8,94 \\
\hline & $--\overline{1}$ & 0 & 0 & 0 & $\overline{0}$ & $-1 \overline{1}, 63$ \\
\hline & 1 & 0 & 0 & 1 & 0 & 21,34 \\
\hline & 1 & 0 & 0 & 0 & 1 & 17,92 \\
\hline & 1 & 0 & 0 & 1 & 1 & 30,17 \\
\hline \multirow{9}{*}{$\begin{array}{l}\text { Faculdade de } \\
\text { Educação }\end{array}$} & $\overline{0}$ & 0 & 0 & 0 & 0 & 13,44 \\
\hline & 0 & 0 & 1 & 0 & 0 & 11,58 \\
\hline & 0 & 1 & 0 & 0 & 0 & 31,31 \\
\hline & 0 & 0 & 0 & 1 & 0 & 15,45 \\
\hline & $\underline{0}$ & 0 & 0 & 0 & 1 & 18,17 \\
\hline & & 0 & 0 & 0 & 0 & $2 \overline{9}, 9 \overline{8}$ \\
\hline & 1 & 0 & 0 & 1 & 0 & 33,12 \\
\hline & 1 & 0 & 0 & 0 & 1 & 37,13 \\
\hline & 1 & 0 & 0 & 1 & 1 & 40,52 \\
\hline \multirow{8}{*}{$\begin{array}{c}\text { Faculdade de } \\
\text { Economia, } \\
\text { Administração, } \\
\text { Atuária, } \\
\text { Contabilidade e } \\
\text { Secretariado }\end{array}$} & $\overline{0}$ & $\overline{0}$ & 0 & 0 & 0 & 17,54 \\
\hline & 0 & 0 & 1 & 0 & 0 & 15,24 \\
\hline & 0 & 1 & 0 & 0 & 0 & 36,99 \\
\hline & 0 & 0 & 0 & 1 & 0 & 22,00 \\
\hline & $-\frac{0}{1}$ & 0 & 0 & 0 & 1 & - - $-22,09$ \\
\hline & $\overline{1}$ & 0 & 0 & $0_{1}^{-}$ & $0^{-}$ & $\begin{array}{r}--3 \overline{3}, 8 \overline{0}--- \\
3986\end{array}$ \\
\hline & 1 & $\begin{array}{l}0 \\
0\end{array}$ & 0 & $\begin{array}{l}1 \\
0\end{array}$ & 1 & $\begin{array}{l}39,80 \\
39,98\end{array}$ \\
\hline & 1 & 0 & 0 & 1 & 1 & 46,29 \\
\hline
\end{tabular}




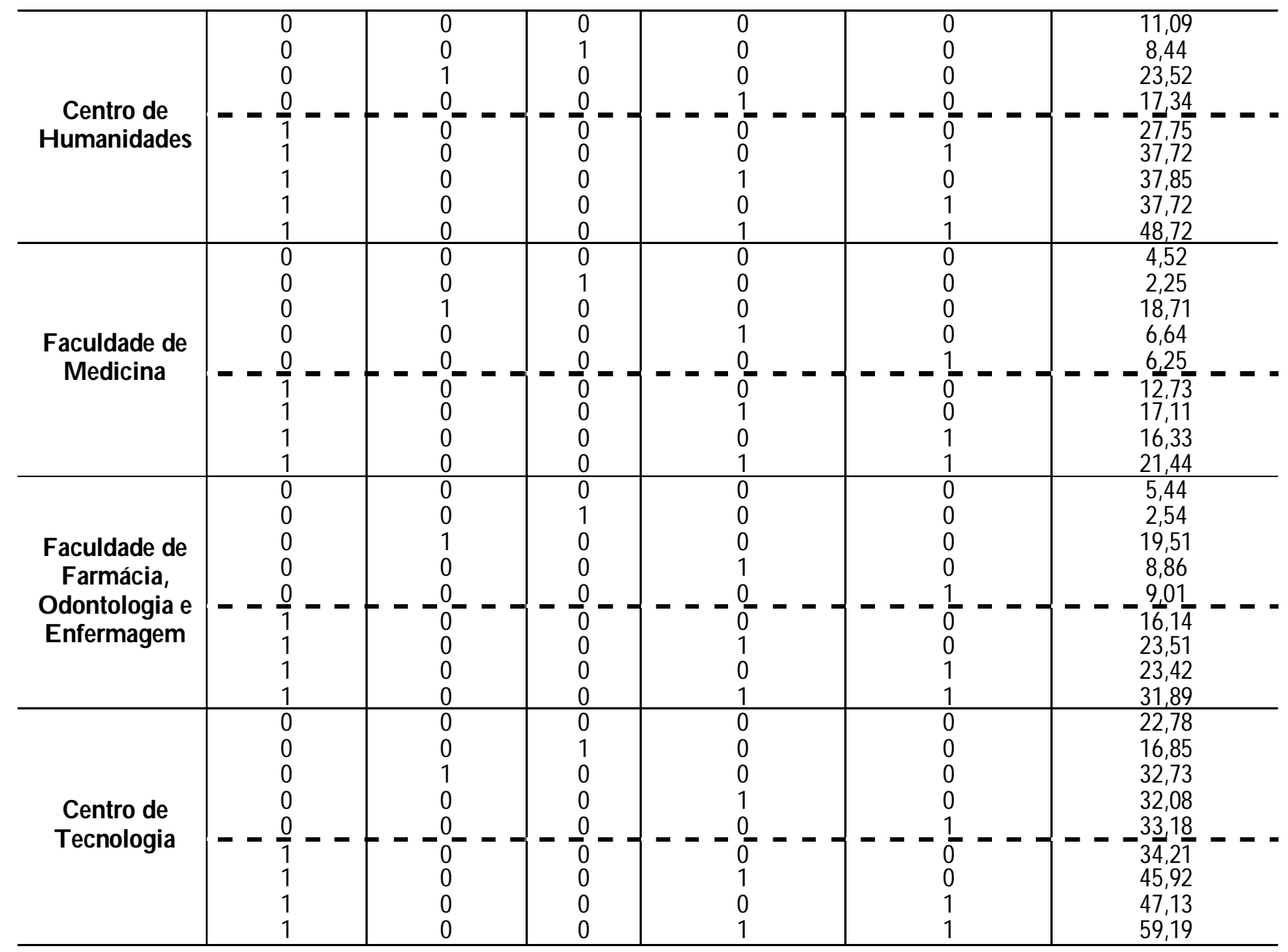

Fonte: Elaborado pelos autores, com base nos dados da CCV.

Percebe-se pela Tabela 1 que ao se trocar o valor zero pelo valor uma probabilidade do candidato obter uma nota acima da nota média da sua referida área aumenta, exceto para a variável trabalho, pois, como já relatado, essa variável reduz tal probabilidade à medida que os valores da variável explicativa aumentam. Como todas as variáveis explicativas são dicotômicas, o simples fato de se alterar o valor de qualquer uma dessas variáveis, coteisparibus retrata o efeito sobre a variação na probabilidade em determinada classe, ou seja, $\operatorname{Pr}\left(Y_{\mathrm{i}}=\boldsymbol{j}\right)$.

Pode-se constatar que as áreas de medicina, direito e odontologia apresentam as menores probabilidades entre todas as áreas, indicando que as características socioeconômicas aqui analisadas apresentam uma menor participação no desempenho de um candidato. Isso possivelmente ocorre em virtude de determinados fatores não incorporados na análise, tais como horas de estudo, capacidade de aprendizagem, entre outros, responderem pela maior participação no desempenho de um candidato dessas três áreas. Por outro lado, pode-se constatar que nas áreas de ciências e tecnologia, as variáveis utilizadas apresentam um forte impacto no desempenho dos candidatos.

A magnitude do efeito marginal que as variáveis exercem sobre a probabilidade de desempenho de um candidato varia entre os centros/ faculdades. $\mathrm{O}$ fato de um candidato possuir uma determinada caracteństica pode ter uma forte influência no seu desempenho em algumas áreas, mas pode apresentar uma fraca influência em outras. Como exemplo, o fato de um candidato ter cursado o ensino médio em escola particular acarreta um efeito marginal positivo de 22,14\% na probabilidade de ele apresentar nota superior ao rendimento médio no Centro de Tecnologia; já na Faculdade de Medicina, tal contribuição é reduzida para 8,2\%. Na possibilidade de que esse fato seja devido à concorrência entre as áreas, fez-se um teste da correlação entre o número de candidatos por vaga e a pontuação média entre as áreas, resultando em um valor estatisticamente não significante de 0,39 . Conclui-se, assim, que concorrência não dita o desempenho dos candidatos no vestibular.

\subsection{Resultados do Modelo Policotômico}

As estimações do modelo probit ordenado mostraramse estatisticamente robustas, vez que os coeficientes de todas as variáveis foram, individualmente e conjuntamente, significantes, além de atenderem às expectativas dos efeitos esperados (ver Tabela A10 no anexo).

Como foi dito anteriormente, tem-se que, majorandose a variável trabalho, a probabilidade de 0 candidato perten- 
cer ao grupo dos piores rendimentos aumenta. Já para as demais variáveis, tem-se que, aumentando-se qualquer uma delas, a probabilidade de 0 candidato pertencer a esse grupo diminui. Para o grupo dos melhores rendimentos tem-se exatamente o oposto, ou seja, uma majoração na variável trabalho reduz a probabilidade de 0 candidato pertencer a esse grupo, e uma elevação em qualquer uma das demais variáveis aumenta essa probabilidade. A Tabela 2 descreve as mudanças que as variações nas variáveis acarretarão nas probabilidades dos três grupos, em cada um dos quais são denotados dois referenciais de cenários separados pela linha tracejada. é um fator decisivo para ditar o desempenho dos candidatos. Nesse sentido, inspecionando a segunda linha desses grupos em que os candidatos trabalham, mantidos os demais fatores iguais, a chance para a elevação de pontuação reduz de 87,7\% para apenas $9,8 \%$. Similarmente, a origem escolar também é incisiva para um candidato ter chance de obter elevada pontuação. O bservando a primeira linha dos dois grupos em referência, confirma-se que a probabilidade de um candidato que frequentou escola pública passar do grupo 2 (desempenho intermediário) para o grupo 3 (maior desempenho) diminui de $82,7 \%$ para $16,1 \%$. Há, nesse sentido, uma forte

Tabela 2: Cenários Probabilísticos

\begin{tabular}{|c|c|c|c|c|c|c|}
\hline Grupos & Ensino Médio & Noo de Vezes & Trabalho & Instrução do Pai & Instrução da Mãe & Probabilidades(\%) \\
\hline \multirow{9}{*}{$\begin{array}{l}\text { Grupo 1: } \\
Y_{i}=0 \\
Y_{i}^{0} \leq 80\end{array}$} & 0 & 0 & 0 & 0 & 0 & 1,17 \\
\hline & 0 & 0 & 1 & 0 & 0 & 2,49 \\
\hline & 0 & 1 & 0 & 0 & 0 & 0,98 \\
\hline & 0 & 0 & 0 & 1 & 0 & 0,29 \\
\hline & 0 & 0 & 0 & 0 & 1 & 0.32 \\
\hline & 1 & $\overline{0}$ & $\overline{0}$ & 0 & $0^{-}$ & $\overline{0,39}--$ \\
\hline & 1 & 0 & 0 & 1 & 0 & 0,08 \\
\hline & 1 & 0 & 0 & 0 & 1 & 0,09 \\
\hline & 1 & 0 & 0 & 1 & 1 & 0,01 \\
\hline \multirow{9}{*}{$\begin{array}{c}\text { Grupo 2: } \\
Y_{i}=1 \\
80<Y_{i}^{0} \leq 160\end{array}$} & 0 & 0 & 0 & 0 & 0 & 82,67 \\
\hline & 0 & 0 & 1 & 0 & 0 & 87,71 \\
\hline & 0 & 1 & 0 & 0 & 0 & 81,18 \\
\hline & 0 & 0 & 0 & 1 & 0 & 68,79 \\
\hline & 0 & 0 & 0 & 0 & 1 & 69,80 \\
\hline & 1 & $\overline{0}$ & $\overline{0}$ & 0 & 0 & $-\overline{72, \overline{1} 1}=$ \\
\hline & 1 & 0 & 0 & 1 & 0 & 54,21 \\
\hline & 1 & 0 & 0 & 0 & 1 & 55,38 \\
\hline & 1 & 0 & 0 & 1 & 1 & 36,21 \\
\hline \multirow{9}{*}{$\begin{array}{l}\text { Grupo 3: } \\
Y_{i}=2 \\
Y_{i}^{0}>160\end{array}$} & 0 & 0 & 0 & 0 & 0 & 16,15 \\
\hline & 0 & 0 & 1 & 0 & 0 & 9,79 \\
\hline & 0 & 1 & 0 & 0 & 0 & 17,83 \\
\hline & 0 & 0 & 0 & 1 & 0 & 30,91 \\
\hline & 0 & 0 & 0 & 0 & 1 & 29,87 \\
\hline & 1 & $\overline{0}$ & $\overline{0}$ & 0 & 0 & $\overline{2} 7, \overline{4} 9^{-}--$ \\
\hline & 1 & 0 & 0 & 1 & 0 & 45,70 \\
\hline & 1 & 0 & 0 & 0 & 1 & 44,52 \\
\hline & 1 & 0 & 0 & 1 & 1 & 63,77 \\
\hline
\end{tabular}

Fonte: Elaborado pelos autores, com base nos dados da CCV.

Ao se observar as características socioeconômicas dos candidatos, constata-se que as maiores probabilidades ocorrem entre aqueles que pertencem ao grupo intermediário, independentemente dos cenários propostos.

As variáveis referentes a trabalho (dedicação exclusiva ou não ao estudo) e tipo de escola que frequentou no ensino médio denotam efeitos mais fortes sobre as performances dos candidatos. Embora com efeito menor, os níveis de instrução do pai e da mãe também representam impactos relevantes nas probabilidades de os candidatos pertencerem ao grupo de melhor desempenho.

Concentrando a análise nos grupos $Y=1$ e $Y=2$, extraise inicialmente que a falta de dedicação integral aos estudos conotação de política pública quanto à qualidade do ensino de escolas públicas.

Contrastando candidatos graduados no ensino médio em escolas particulares, seus badkgrands familiares também exercem significante influência sobre suas performances na pontuação do vestibular. Examinando a última linha dos dois grupos, em que os pais possuem nível de ensino superior, verifica-se que a diferença das probabilidades é de cerca de 27 pontos percentuais em favor do grupo de pontuação mais elevada. Nesse aspecto, percebe-se um equilíbrio de influência dos níveis educacionais do pai e da mãe do candidato, conforme os resultados da sétima e oitava linha em ambos os grupos. 
Dos resultados para $Y=2$, conclui-se que candidatos que maximizam a chance de obtenção de êxito em cursos que exibem as maiores pontuações, tais como medicina, odontologia e as engenharias, enquadram-se em um perfil caracterizado por dedicação integral aos estudos, cursaram o ensino médio em escolas particulares e possuem pais com formação universitária.

\section{CONCLUSÕES}

Das estimações dos modelos para se inferir sobre as chances de sucesso de candidatos ao vestibular da Universidade Federal do Ceará (UFC), conclui-se que as estimativas apresentaram-se bastante homogêneas entre os modelos alternativos propostos, indicando consistência para as conclusões deles extraídas. Conclui-se também que, apesar da metodologia distinta aqui empregada, os resultados obtidos foram compatíveis com os obtidos em outros trabalhos correlatos, comparando-se os efeitos de algumas variáveis comuns. Reconhece-se, todavia, que algumas variáveis afetas aos candidatos, as quais poderiam contribuir para explicar os rendimentos deles no concurso, tais como raça, renda familiar e horas de estudo, não se dispunham no banco de dados. Não obstante, as demais se mostraram eficazes e com forte poder de explicação no fenômeno investigado, satisfazendo, assim, o objetivo do estudo.

Constatou-se que os rendimentos dos candidatos ao vestibular que estudaram em escolas particulares são superiores aos daqueles que estudaram em escolas públicas. $\mathrm{Ou}$ seja, a probabilidade de estar no grupo dos candidatos com melhores notas é maior entre aqueles que estudaram em escola particular. Embora esse resultado seja esperado, pelo que se tem preconizado de há muito sobre o ensino de escolas públicas, ratifica-se a omissão de governos em prover educação com qualidade.

Sobre o badkgrand familiar, comprovou-se que, no geral, há prevalência de efeitos positivos sobre rendimentos dos candidatos no concurso de vestibular, significando que candidatos de pais com nível superior apresentam maior probabilidade de se situarem no grupo daqueles com maior nota. Essa conclusão se estende para aqueles candidatos que possuam apenas o pai ou a mãe com nível educacional superior. Ressalve-se que, em um dos modelos analisados, a titulação do pai apresentou um impacto levemente superior ao da titulação da mãe na probabilidade do candidato pertencer ao grupo de melhor desempenho no geral. Já na análise entre áreas, realizada em outro modelo, algumas áreas, como agrárias, ciências e educação mostraram que a titulação da mãe apresenta um maior impacto, ao passo que em áreas como direito e medicina mostraram que é a titulação do pai que apresenta um maior impacto.

$\mathrm{O}$ fato de um candidato trabalhar durante a sua vida escolar afeta sobremaneira o seu rendimento no vestibular, ou seja, indivíduos que trabalham apresentam um rendimento pior e consequentemente maior será a probabilidade de se situarem no grupo dos candidatos com as piores notas.
Em relação à repetência no concurso de vestibular, parece que a experiência dos anos anteriores proporciona um melhor rendimento aos candidatos; portanto, chega-se à conclusão de que candidatos com maiores experiências nesse exame de vestibular apresentam a probabilidade de um melhor desempenho.

Quanto às áreas presentes no vestibular da UFC, deduz-se que algumas delas apresentam um maior grau de dificuldade para pertencer ao grupo de melhor desempenho. Candidatos de áreas diferentes, e que apresentam as mesmas características, obtêm probabilidades distintas de pertencer ao grupo de desempenho elevado nas suas áreas de escolha e, em alguns casos, a discrepância nos resultados chega a ser significativa, como se constata, por exemplo, ao se comparar as áreas de medicina e tecnologia.

\section{REFERÊNCIAS}

ALBERNAZ, A.; FERREIRA, F.; FRANCO, C. Q ualidade e equidade no ensino fundamental brasileiro. Pequisa e Plangamento Econômico v. 32, n.3, 2002.

ASPLUND, R.; et al. NaEquityPespeetiveonA cessto Enrdmettinand Finance of Tetiary Eduration D isponível em: <www.etla.fifiles1821 D 1098.pdf>. Acesso em: 22 maio 2008.

AVENA, Cláudio Pondé. Demanda por ensino superior a partir deuma abordaggmob capital humano D isponível em: <http:/ / www.anpec.org. br/ encontro2003/ artigos/ F29.pdf>. Acesso em: 01 maio 2008.

CO STA, L. O. ARRAES, R. Contrastes da Pdítica Educadional Brasilera entre Escolas Públicas e Privadas. Disponível em: <http:/ / www. sustentavel.inf.br/ anexos/ publicacao/ konrad_desigualdades_e_ politicas_regionais.pdf $>$. Acesso em: 23 maio 2008.

DE FELÍCIO, F.; FERNANDES, R. O Efäto da Qualidadeda Escda sobre o Desempenho Escdar: Uma Avaliação do Ensino Fundamental no Estado de São Paulo. D isponível em: < http:/ / www.anpec.org.br/ encontro2005/ artigos/ A05A157.pdf>. A cesso em: 21 abr. 2008.

DAVID SON, Russel; MACKINNON, James G. Econmenic Thery and Methods New York: Oxford University Press, 2004.

DUCZMAL, Luis H.; PEREIRA, L. A. dos Santos. Análise das mensalidades de algumas esclas partiaulares de Bdo Honizonte e desernpenho no vestibular. D isponível em: <ftp:/ / ftp.est.ufmg.br/ pub/ rts/ rta0301.pdf >. Acesso em: 21 abr. 2008.

DUMAS, Christelle; LAMBERT Sylvie. Educational Adievement and SociceonomicBadkgrand: CausalityandMehanisnsin Senegal. D isponivel em: <http:/ / www.inra.fr/ internet/ D epartements/ ESR/ UR/ lea/ documents/wp/ wp0706.pdf>. Acesso em: 21 set. 2007.

EHREMBERG, Ronald G.; SMITH, Robert S. A Modema Economia db Trabalha Teoria e Política Pública. 5. ed., São Paulo: MAKRON Books, 2000.

FRENETTE, Marc; ZEMAN, Klarka. Why Are Most University Studants Women? Evidance Based on Academic Peformane, Study Habits and Parental Influences. Disponível em: <http:/ / www.statcan. caenglishresearch11F0019MIE 11F0019MIE2007303.pdf >. Acesso em: 21 set. 2007.

GREENE, William H. Econamenic Analysis 3. ed., New Jersey: Prentice Hall, 2000.G UIMARÃES, Antonio Sérgio Alfredo. Ingesso emunivesidades deprestígo edesempenho movestibular degupos privilegjados negativamente negros na Universidade de São Paulo, de 2001 a 2007. Disponível em: <http:/ / www.fflch.usp.br/ sociologiaasagnegros\% 
20na\%20universidade \%20de $\% 20$ S\%C3\%A 30\%20Paulo \%20de\% 202001\%20a\% 202007.pdf>. Acesso em: 21 abr. 2008.

G UIMARÃES, Juliana; SAMPAIO, Breno. The Influence of Family BadkgaundandIndvidual CharaderisticsonEntranceTestsScores of Brazilian University Students. D isponível em: <http:/ / www.anpec.org. brencontro2007artigosA 07A 092.pdf. Acesso em: 21 abr. 2008.

G UJARATI, D amondar N. Economeria Básica. 3. ed., São Paulo: MAKRON Books, 2000.

HANUSHEK, A. E. The Economics of Schooling: Production and Efficiency in Public Schools. Jaumal of Econamic Literature n. 24, p. 1141-1177, 1986.

INEP. Censoda EducaçãoSuperior. D isponível em: < http:/ / www.inep. gov.br/ superior/ censosuperior/ >. Acesso em: 01 maio 2008.

KAPUR, D.; CROWLEY, M. Beyond theABCs: Higher Education and DedapingCantries D isponível em: < http:/ / www.policypointers.org/ page_7102.html>. Acesso em: 23 maio 2008.

MADDALA, G. S. Introduction to Econametrics. 2. ed., New York: Macmillan Published Company, 1988.

QUEIROZ, D elcele Mascarenhas. DesigualdadenoEnsinoSuperior. Cor, Status e D esempenho. D isponível em: <http:/ / www.anped.org. brreunioes26trabalhosdelcelemascarenhasqueiroz.pdf $>$. A cesso em: 21 fev. 2008.

SAKS, Raven; SHO RE, Stephen H. Risk and career dhice D isponível em: <http:/ / kuznets.fas.harvard.edu/ rsaks/ papers/ career503. pdf $>$. Acesso em: 21 jun. 2005.

SOARES, J. F. Q ualidade e equidade na educação básica Brasileira: A evidência do SAEB-2001. Arquivos Analíticos de Políticas Educativas. D isponível em: <http:/ / epaa.asu.edu/ epaa/ v12n38>. Acesso em: 31 maio 2008.

VELLO SO, Jacques. Curso econarsa rendimento na universidade e desempenho em um vestibular com cotas da UnB. D isponível em: <http:/ / www.ifcs.ufrj.br observarelatoriosCursoeconcurso_UnB. pdf>. Acesso em: 21 abr. 2008.

WO O LD RID G E, Jeffrey M. EconmenicAmalysis of Cross Section and Pand Data. London: The MIT Press, 2002.

\section{NOTAS DE FIM DE TEXTO:}

${ }^{1}$ A definição de sucesso nos termos deste trabalho refere-se ao desempenho do candidato no vestibular;

${ }^{2}$ As estimações das equações que geraram os cenários discutidos nesta seção estão dispostas nas Tabelas A.1 a A.9 do anexo.

Data de Submissão: 22/ 10/ 2009

Data de Aprovação: 25/ 07/ 2010 


\section{ANEXO}

Tabela A.1: Estimativas do Modelo Dicotômico para a Área de Agrárias

\begin{tabular}{ccccc}
\hline Variáveis & Coeficientes & Erro Padrão & Estatítica z & Valor P \\
\hline C & -0.925450 & 0.040372 & -22.92313 & 0.0000 \\
ENS_MEDIO & 0.468964 & 0.045618 & 10.28024 & 0.0000 \\
N_VEZES & 0.561056 & 0.042049 & 13.34284 & 0.0000 \\
TRABALHO & -0.141386 & 0.050562 & -2.796305 & 0.0052 \\
INST_PAI & 0.190824 & 0.060816 & 3.137713 & 0.0017 \\
INST_MAE & 0.204144 & 0.057855 & 3.528514 & 0.0004 \\
\hline Log Verossimilhança & & & -2487.559 & \\
Razão de Verossimilhança (5 gl) & & & 457.8296 & \\
Valor P & 2635 & Total de O bservações & & \\
\hline Obs com Dep=0 & 1508 & & & \\
Obs com Dep=1 & & & \\
\hline
\end{tabular}

Fonte: Elaborado pelos autores, com base nos dados da CCV.

Tabela A.2: Estimativas do Modelo Dicotômico para a Área de Ciências

\begin{tabular}{ccccc}
\hline \multicolumn{1}{c}{ Variáveis } & Coeficientes & Erro Padrão & Estatítica z & Valor P \\
\hline C & -0.126298 & 0.032749 & -3.856599 & 0.0001 \\
ENS_MEDIO & 0.520174 & 0.043226 & 12.03374 & 0.0000 \\
N_VEZES & 0.505164 & 0.041022 & 12.31452 & 0.0000 \\
TRABALHO & -0.097723 & 0.043938 & -2.224090 & 0.0261 \\
INST_PAI & 0.178680 & 0.065516 & 2.727256 & 0.0064 \\
INST_MAE & 0.332421 & 0.066331 & 5.011512 & 0.0000 \\
\hline Log Verossimilhança & & & -2785.972 & \\
Razão de Verossimilhança (5 gl) & & & 513.3042 & \\
Valor P & 1701 & Total de observações & & \\
\hline Obs com Dep=0 & 2925 & & & \\
Obs com Dep=1 & & &
\end{tabular}

Fonte: Elaborado pelos autores, com base nos dados da CCV.

Tabela A.3: Estimativas do Modelo D icotômico para a Área de Direito

\begin{tabular}{|c|c|c|c|c|}
\hline Variáveis & Coeficientes & Erro Padrão & Estatítica z & Valor P \\
\hline $\mathrm{C}$ & -1.619431 & 0.070957 & -22.82281 & 0.0000 \\
\hline ENS_MEDIO & 0.426094 & 0.070221 & 6.067859 & 0.0000 \\
\hline N_VEZES & 0.561898 & 0.055091 & 10.19944 & 0.0000 \\
\hline TRABALHO & -0.199217 & 0.072219 & -2.758524 & 0.0058 \\
\hline INST_PAI & 0.398878 & 0.061424 & 6.493905 & 0.0000 \\
\hline INST_MAE & -1.619431 & 0.070957 & -22.82281 & 0.0000 \\
\hline Log Verossimilhança & & & -1485.127 & \\
\hline Razão de Verossimilhança (5 gl) & & & 341.1996 & \\
\hline Valor P & & & 0.000000 & \\
\hline Obs com Dep $=0$ & 2589 & Total de observações & & 3259 \\
\hline Obs com Dep=1 & 670 & & & \\
\hline
\end{tabular}

Fonte: Elaborado pelos autores, com base nos dados da CCV. 
Tabela A.4: Estimativas do Modelo Dicotômico para a Área de Educação

\begin{tabular}{|c|c|c|c|c|}
\hline Variáveis & Coeficientes & Erro Padrão & Estatítica z & Valor P \\
\hline $\mathrm{C}$ & -1.105584 & 0.050295 & -21.98194 & 0.0000 \\
\hline ENS_MEDIO & 0.580685 & 0.062260 & 9.326752 & 0.0000 \\
\hline N_VEZES & 0.618544 & 0.058757 & 10.52707 & 0.0000 \\
\hline TRABALHO & -0.090751 & 0.063306 & -1.433527 & 0.1517 \\
\hline INST PAI & 0.088411 & 0.095566 & 0.925126 & 0.3549 \\
\hline INST_MAE & 0.196663 & 0.088857 & 2.213254 & 0.0269 \\
\hline Log Verossimilhança & & & -1315.294 & \\
\hline Razão de Verossimilhança ( 5 gl) & & & 296.3752 & \\
\hline Valor P & & & 0.000000 & \\
\hline Obs com Dep=0 & 1809 & Total de O bservações & & 2492 \\
\hline Obs com Dep=1 & 683 & & & \\
\hline
\end{tabular}

Fonte: Elaborado pelos autores, com base nos dados da CCV.

Tabela A.5: Estimativas do Modelo Dicotômico para a Área da Faculdade de Economia, Administração, Atuária, Contabilidade e Secretariado

\begin{tabular}{ccccc}
\hline \multicolumn{1}{c}{ Variáveis } & Coeficientes & Erro Padrão & Estatítica z & Valor P \\
\hline C & -0.932846 & 0.037319 & -24.99646 & 0.0000 \\
ENS_MEDIO & 0.515136 & 0.041918 & 12.28901 & 0.0000 \\
N_VEZES & 0.600839 & 0.039041 & 15.39011 & 0.0000 \\
TRABALHO & -0.093031 & 0.041447 & -2.244582 & 0.0248 \\
INST_PAI & 0.160797 & 0.057966 & 2.773984 & 0.0055 \\
INST_MAE & 0.164016 & 0.057294 & 2.862712 & 0.0042 \\
\hline Log Verossimilhança & & & -2939.008 & \\
Razão de Verossimilhança (5 gl) & & & 580.7432 & \\
Valor P & 3215 & Total de O bservações & & \\
\hline Obs com Dep=0 & 1757 & & & \\
Obs com Dep=1 & & & & \\
\hline
\end{tabular}

Fonte: Elaborado pelos autores, com base nos dados da CCV.

Tabela A.6: Estimativas do Modelo Dicotômico para a Área de Humanidades

\begin{tabular}{ccccc}
\hline Variáveis & Coeficientes & Erro Padrão & Estatítica z & Valor P \\
\hline C & -1.221682 & 0.031002 & -39.40665 & 0.0000 \\
ENS_MEDIO & 0.631549 & 0.036054 & 17.51665 & 0.0000 \\
N_VEZES & 0.500060 & 0.033746 & 14.81831 & 0.0000 \\
TRABALHO & -0.154250 & 0.039555 & -3.899594 & 0.0001 \\
INST_PAI & 0.280745 & 0.047042 & 5.968012 & 0.0000 \\
INST_MAE & 0.277458 & 0.045529 & 6.094053 & 0.0000 \\
\hline Log Verossimilhança & & & -3888.631 & \\
Razão de Verossimilhança (5 gl) & & & 0.000000 & \\
Valor P & 5555 & Total de Observações & & \\
\hline Obs com Dep=0 & 2024 & & & \\
Obs com Dep=1 & &
\end{tabular}

Fonte: Elaborado pelos autores, com base nos dados da CCV. 
Tabela A.7: Estimativas do Modelo Dicotômico para a Área de Medicina

\begin{tabular}{ccccc}
\hline \multicolumn{1}{c}{ Variáveis } & Coeficientes & Erro Padrão & Estatítica z & Valor P \\
\hline C & -1.692614 & 0.082487 & -20.51967 & 0.0000 \\
ENS_MEDIO & 0.553364 & 0.082041 & 6.744938 & 0.0000 \\
N_VEZES & 0.804121 & 0.049580 & 16.21881 & 0.0000 \\
TRABALHO & -0.311838 & 0.094119 & -3.313246 & 0.0009 \\
INST_PAI & 0.189766 & 0.054658 & 3.471892 & 0.0005 \\
INST_MAE & 0.158575 & 0.055026 & 2.881822 & 0.0040 \\
\hline Log Verossimilhança & & & -1834.197 & \\
Razão de Verossimilhança (5 gl) & & & & \\
Valor P & 2536 & Total de Observações & & 3511 \\
\hline Obs com D ep=0 & 975 & & & \\
Obs com D ep=1 & & & & \\
\hline
\end{tabular}

Fonte: Elaborado pelos autores, com base nos dados da CCV.

Tabela A.8: Estimativas do Modelo Dicotômico para a Área da Faculdade de O dontologia, Farmácia e Enfermagem

\begin{tabular}{ccccc}
\hline Variáveis & Coeficientes & Erro Padrão & Estatítica z & Valor P \\
\hline C & -1.603692 & 0.058586 & -27.37324 & 0.0000 \\
ENS_MEDIO & 0.615191 & 0.061932 & 9.933404 & 0.0000 \\
N_VEZES & 0.744617 & 0.047558 & 15.65699 & 0.0000 \\
TRABALHO & -0.349213 & 0.077208 & -4.523024 & 0.0000 \\
INST_PAI & 0.254424 & 0.056032 & 4.540705 & 0.0000 \\
INST_MAE & 0.263553 & 0.054682 & 4.819746 & 0.0000 \\
\hline Log Verossimilhança & & & -1862.518 & \\
Razão de Verossimilhança (5 gl) & & & 653.6324 & \\
Valor P & 2996 & Total de Observações & & \\
\hline Obs com Dep=0 & 958 & & & \\
Obs com Dep=1 & & & & \\
\hline
\end{tabular}

Fonte: Elaborado pelos autores, com base nos dados da CCV.

Tabela A.9: Estimativas do Modelo Dicotômico para a Área de Tecnologia

\begin{tabular}{ccccc}
\hline Variáveis & Coeficientes & Erro Padrão & Estatítica z & Valor P \\
\hline C & -0.769560 & 0.046955 & -16.38948 & 0.0000 \\
ENS_MEDIO & 0.362950 & 0.053134 & 6.830876 & 0.0000 \\
N_VEZES & 0.322172 & 0.046983 & 6.857212 & 0.0000 \\
TRABALHO & -0.190382 & 0.055136 & -3.452969 & 0.0006 \\
INST_PAI & 0.304342 & 0.056989 & 5.340368 & 0.0000 \\
INST_MAE & 0.334818 & 0.056520 & 5.923840 & 0.0000 \\
\hline Log Verossimilhança & & & -2097.100 & 348.0157 \\
Razão de Verossimilhança (5 gl) & & & 0.000000 & \\
Valor P & 2063 & & \\
\hline Obs com Dep=0 & 1329 & Total de Observações & & \\
Obs com Dep=1 & & & & \\
\hline
\end{tabular}

Fonte: Elaborado pelos autores, com base nos dados da CCV. 
Tabela A.10 - Estimativas do Modelo Policotômico

\begin{tabular}{ccccc}
\hline Variáveis & Coeficientes & Erro Padrão & Estatística Z & Valor P \\
\hline ENSINO_MEDIO & 0.390271 & 0.030765 & 12.68540 & 0.0000 \\
N_VEZES & 0.066614 & 0.025861 & 2.575844 & 0.0100 \\
TRABALHO & -0.304980 & 0.032709 & -9.323931 & 0.0000 \\
INST_PAI & 0.489953 & 0.030899 & 15.85647 & 0.0000 \\
INST_MAE & 0.460291 & 0.030658 & 15.01392 & 0.0000 \\
\hline \multicolumn{2}{c}{ Pontos Limites } & & \\
\hline Limite_1:C(6) & -2.266366 & 0.051183 & -44.27954 & 0.0000 \\
Limite_2:C(7) & 0.988189 & 0.031327 & 31.54443 & 0.0000 \\
\hline Log Verossimilhança & & & -6631.918 \\
Razão de Verossimilhança (5 gl) & & & & 1751.317 \\
Valor P & & & & 0.000000 \\
\hline
\end{tabular}

Fonte: Elaborado pelos autores, com base nos dados da CCV. 